\title{
Study on the Stability of CFAEs to Characterize the Atrial Substrate in Atrial Fibrillation
}

\author{
Emanuela Finotti ${ }^{1}$, Edward J Ciaccio ${ }^{2}$, Hasan Garan ${ }^{2}$, \\ Fernando Hornero ${ }^{3}$, Raúl Alcaraz ${ }^{4}$, José J Rieta ${ }^{1 *}$ \\ ${ }^{1}$ BioMIT.org, Electronic Engineering Department, Universitat Politecnica de Valencia, Spain \\ ${ }^{2}$ Department of Medicine - Division of Cardiology, Columbia University, New York, USA \\ ${ }^{3}$ Cardiovascular Surgery Department, Hospital Clínico Universitario de Valencia, Valencia, Spain \\ ${ }^{4}$ Research Group in Electronic, Biomed. and Telecomm. Eng., Univ. of Castilla-La Mancha, Spain
}

\begin{abstract}
A variety of indexes has been applied to complex fractionated atrial electrograms (CFAEs) of atrial fibrillation $(A F)$ aimed at characterizing the atrial substrate. However, often the reported results miss the assessment of intra-recording and intra-patient stability of the analyzed data, as well as CFAEs signal quality. This work introduces a study in which Determinism (DET) of Recurrence Quantification Analysis (RQA) and Sample Entropy (SE) have been applied to assess intra-recording and intrapatient stability of 1, 2 and 4 s-length segments CFAEs recorded from patients with paroxysmal and persistent $A F$ using the coefficient of variation $(C V)$. Furthermore, the analyses verified changes introduced by discarding artifacted and noisy CFAE segments. The intra-recording analysis pointed out that discarding segments provoked a significant variation of $\mathrm{CV}(\%)$ in any segment length both for DET and SE, with deeper decreases for longer segments. Intra-patient stability provided large variations in $C V(\%)$ for DET and even bigger for SE at any segment length. In this case discarding segments was useless and $C V$ provided limited variations. Kruskal-Wallis test revealed significant differences in DET and SE values among channels, independently from the discarding process.
\end{abstract}

\section{Introduction}

Atrial fibrillation (AF) is the most common arrhythmia diagnosed in clinical practice, with estimated prevalence of about $1-2 \%$ of the population worldwide [1]. Thanks to the long-term success observed in some patients, catheter ablation is considered the first line alternative to heart rate control medications. Thus, many research efforts in AF are addressed in mapping the atrial electrophysiological substrate to identify the ectopic sites responsible of propagating the abnormal electrical impulses. In the attempt of personalizing the AF treatment, non-linear indexes are often applied to complex fractionated atrial electrograms (CFAEs) to quantify the atrial remodeling, support clinical management decisions and suggest the most appropriate approach for ablation procedures.

In the literature can be found many works in which nonlinear indexes were applied to invasive electrograms: E. J. Ciaccio et al. measured CFAEs repetitiveness [2] and quantified the degree of morphological heterogeneity in CFAE deflections [3]; U. R. Acharya et al. adopted recurrence plots, RQA and entropy measures [4]; G. Ndrepepa et al. and K. C. Ravi et al. used the atrial fibrillation cycle length [5] [6]; P. Sanders et al. employed spectral analysis and the dominant frequency mapping [7].

However, often the reported results miss the assessment of intra-recording and intra-patient stability of the analyzed data, as well as CFAEs signal quality. With these omissions, two main issues may arise: first, averaging among measurement sites and AF types without having previously checked the intra-recording and intra-patient stability may lead to an oversimplification of the processes taking place at different regions of the left atrium; second, the inclusion of artifacted and noisy segments unlinked to the AF mechanism, such as drifts and zero amplitudes sequences, may lead to biased and unreliable numbers.

The present work introduces a study in which intrarecording and intra-patient stability of CFAEs have been assessed with two non-linear indexes: the Determinism (DET) of Recurrence Quantification Analysis (RQA) and Sample Entropy (SE). The metrics used to quantify the stability is the coefficient of variation (CV), expressed in \%. Furthermore, the presence of artifacted and noisy segments in CFAEs has been considered as well, evaluating the consequences of their discards in the final outcomes.

The hypothesis is that introducing a discarding process to remove poor quality segments may benefit intrarecording and intra-patient stability. Also, this approach 
may decrease the differences in DET and SE between intra-patient channels, thus helping in quantifying the atrial substrate with reliable and representative values.

\section{Material and Methods}

\subsection{Data Preprocessing}

A total number of 204 electrograms of about 16 s were acquired at $977 \mathrm{~Hz}$ from paroxysmal (ParAF) and longstanding persistent (PerAF) AF patients, not on arrhythmogenic drug therapy, who underwent RFCA at the cardiac electrophysiological laboratory of Columbia University Medical Center. For ParAF and PerAF were recorded respectively 90 and 114 CFAEs, identified observing the published criteria [2], from the four pulmonary veins and the anterior/posterior free wall of the left atrium. If $\mathrm{AF}$ was persisting more than $10 \mathrm{~min}$, then the electrogram was included for analysis and subjected to rectangular 1, 2 and 4 second-length windowing function creating three distinct datasets, each containing 204 non-overlapping sequences of different length. The Internal Review Board (IRB) at Columbia University Medical Center approved acquisition and analysis of these retrospective data. In ParAF with a baseline sinus rhythm, AF was induced by rapid pacing at the coronary sinus or at the lateral wall of the right atrium (coupling interval range $250-200 \mathrm{~ms}$ ). Signals were filtered with a bandpass by the acquisition system prior to discretization, which removed baseline drift and high frequency noise (CardioLab, GE Healthcare, Waukesha, WI) [3], then resampled at $1 \mathrm{KHz}$ and filtered once again with wavelet-based and median filters, which respectively removed the muscle noise and the baseline wander. Signal amplitudes were measured in millivolts.

\subsection{Discarding process}

CFAEs were visually inspected to evaluate their signal quality. Initially, the quality was assessed on $1 \mathrm{~s}$-length sequences, as they belong to the smallest window length, and then the goodness of 2 and 4 s-length segments was derived from these by taking mutually exclusive subsets of 2 and 4 sequences of 1 s-length and setting the quality of each group depending on whether it contains just qualitative 1 s-length segments or at least one element to discard. With this method, few good sequences were discarded, causing a loss of information. The subsets were created following the segments' time order, so that there was a time correspondence between datasets of different length.

The CFAEs exploration had two main focuses: distinguishing drifts from atrial activations and identifying those CFAE segments containing just noise. First, to find out drifts the Welch spectrum of the 1 s-length segments were visualized and those segments presenting peaks in the range $0-2 \mathrm{~Hz}$ were observed in the time domain: the segments having at least an atrial activation with abnormal amplitude and/or shape were discarded. Second, to recognize parts of CFAEs with no atrial activation, the amplitude ranges of each 1 s-length sequence was extracted and the segments presenting ranges around zero $\mathrm{mV}$ were discarded. The percentage of discards was assessed for each window length, measurement site and AF type.

\subsection{Sample entropy}

SE was applied to 1, 2 and 4 s-length sequences datasets. The SE index assigned a non-negative value reflecting the complexity of each sequence, with larger values corresponding to more irregularity in the data. The maximum template length was set at 2 and the tolerance at 0.35 times the standard deviation of the segment, as in previous works [8]. The results are reported with range, mean and standard deviation.

\subsection{Recurrence Plots and RQA measures}

Recurrence plots (RPs) were used to visualize recurrent pattern within 1, 2 and 4 s-length sequences. In order to produce RPs from CFAE segments, considered as a time series, their trajectory were projected into a $d$-dimensional phase space (PS). The PS reconstruction was obtained following Takens' theorem by using an embedding dimension $d$ and a time delay $\tau$, respectively found with Find Nearest Neighbors and Mutual Information methods. Next, the RPs were produced representing each pair of trajectory states as a black dot if their distance is below a specified threshold and with a white dot otherwise. The threshold was set at the $10 \%$ of the mean of the PS diameter [9].

The RQA measure selected for the study is the determinism, which quantifies the sequence predictability by measuring the percentage of recurrence points that belong to diagonal lines of a minimum length $l_{\min }>50$. The results are reported with range, mean and standard deviation.

\subsection{Study of CFAEs stability}

The stability of the indexes, both intra-recording and intra-patient, was checked for the 1,2 and 4 s-length datasets, using the coefficient of variation $(\mathrm{CV})$ as a measure of the dispersion. The coefficient of variation, expressed as $\%$, was calculated as $\mathrm{CV}=\sigma / \mu$, with $\sigma$ the standard deviation and $\mu$ the mean of the index under study. The analyses were repeated discarding artifacted or noisy CFAE segments and any increase/decrease in the CV was assessed. Additionally, the intra-patient analysis was completed graphically with box plots reporting the values of the indexes at the different measurement sites and the overall intra-patient average. 


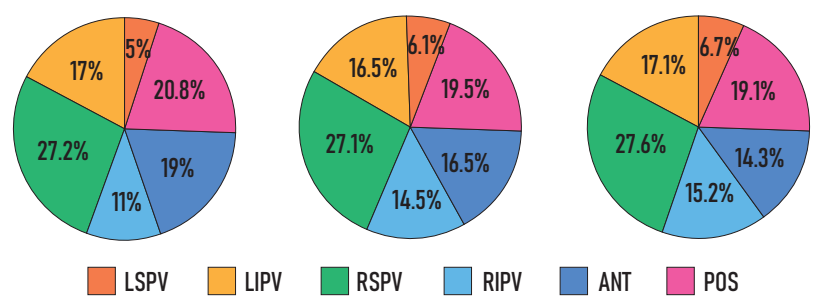

Figure 1. Discarding segments percentages distribution among measurement sites in 1 s-length dataset (A), 2 slength dataset (B) and 4 s-length dataset (C).

Furthermore, the Kruskal-Wallis test was selected to assess whether the index at the different measurement sites of a given patient originate from the same distribution. Prior, the assumption of homokedasticity was verified with the Breush-Pagan test. The method was supported by comparing the median values of the groups both numerically and graphically, so that any inaccuracy could have been detected. The null hypothesis $\left(\mathrm{H}_{0}\right)$ tested was that the mean ranks of DET (or SE) among the intra-patient measurement sites is the same. The acceptance of $\mathrm{H}_{0}$ proved that the atrial remodeling is similar at different sites of the left atrium. Contrarily, the rejection of $\mathrm{H}_{0}$ lead to the conclusion that averaging among channels provoke an oversimplification of the AF mechanisms taking place in the left atrium. The Kruskal-Wallis test was performed on the 1,2 and 4 seconds-length datasets, with and without discards.

\section{Results}

From the CFAEs inspection, the 1-second length segments discarded were the $5.6 \%$ of the totality, while for the 2 and 4 second-length segments the percentages are slightly higher, respectively $8.1 \%$ and $12.9 \%$, due to the additional loss of information. Figure 1 shows the discarded segments distribution along the measurement sites in the datasets. In particular, the numbers of discards in LSPV is quite low as compared with the other channels, in contrast with the RSPV in which the discards are more frequent. The proportion of the discarded segments in paroxysmal and persistent AF patients is similar: for $1 \mathrm{~s}$-length segments, the $46.4 \%$ of the discarded segments were in ParAF and the $53.6 \%$ in PerAF; for 2 s-length segment, the $44.4 \%$ were in ParAF and the $55.6 \%$ in PerAF; for 4 slength segment, the $43.8 \%$ were found to belong to ParAF and the $56.2 \%$ to PerAF.

The averaged statistical descriptors (range, mean, standard deviation) resulted by applying SE to 1, 2 and 4-s length datasets, with and without discards, are reported in table 1. With discards, the ranges were reduced as the lower boundary took greater values due mainly to the remove of drifts, which generally presented high amplitude
Table 1. Sample entropy statistical descriptors analysis results for 1, 2 and 4 s-length segments datasets, with and without discards. The values reported were obtained by averaging among CFAEs.

\begin{tabular}{|c|c|c|c|c|}
\hline & \multicolumn{2}{|c|}{ no discard } & \multicolumn{2}{|c|}{ discard } \\
\hline & range & mean \pm std & range & mean \pm std \\
\hline $1 \mathrm{~s}$ & {$[0.004-0.362]$} & $0.137 \pm 0.033$ & {$[0.013-0.362]$} & $0.138 \pm 0.032$ \\
\hline $2 \mathrm{~s}$ & [0.007-0.312] & $0.134 \pm 0.025$ & [0.018-0.312] & $0.135 \pm 0.023$ \\
\hline $4 s$ & {$[0.008-0.3]$} & $0.132 \pm 0.017$ & {$[0.02-0.3]$} & $0.134 \pm 0.014$ \\
\hline
\end{tabular}

Table 2. Determinism statistical descriptors analysis results for 1, 2 and 4 s-length segments datasets, with and without discards. The values reported were obtained by averaging among CFAEs.

\begin{tabular}{|c|c|c|c|c|}
\hline & \multicolumn{2}{|c|}{ no discard } & \multicolumn{2}{|c|}{ discard } \\
\hline & range & mean \pm std & range & mean \pm std \\
\hline $1 \mathrm{~s}$ & {$[0.075-0.998]$} & $0.561 \pm 0.120$ & {$[0.075-0.963]$} & $0.559 \pm 0.113$ \\
\hline $2 \mathrm{~s}$ & {$[0.122-1]$} & $0.600 \pm 0.101$ & [0.122-0.958] & $0.595 \pm 0.091$ \\
\hline $4 s$ & {$[0.092-1]$} & $0.631 \pm 0.072$ & [0.124-0.942] & $0.621 \pm 0.058$ \\
\hline
\end{tabular}

and low SE values. As a consequence, also the standard deviations decreases with discards.

The statistical descriptors of DET were computed in the same way as SE, and the results are reported in table 2 . For DET, the ranges were reduced with discards, but due to the upper boundary that took lower values, thus decreasing also the standard deviations. In fact, DET and SE are complementary measure as one measure the predictability and the other the complexity.

The intra-recording analysis showed a significant variation of $\mathrm{CV}(\%)$ in any segment length both for DET and $\mathrm{SE}$, as shown in the table 3 . Discarding segments benefited stability decreasing $\mathrm{CV}$ with deeper decreases for longer segments. These variations were bigger in average for PerAF $(\mathrm{DET}=29.1 \%, \mathrm{SE}=37.6 \%)$ than for ParAF $(\mathrm{DET}=19.6 \%, \mathrm{SE}=31.8 \%)$.

Intra-patient stability also provided large variations in $\mathrm{CV}(\%)$ for DET and even bigger for SE at any segment length, as shown in the table 4. In this case discarding segments was useless and CV provided limited variations.

The Kruskal-Wallis test, as well as the visual inspection of the box plots, revealed that the atrial remodeling is mostly different at the measurement sites analyzed showing a great variability of the indexes intra-patient. For 1 s-length datasets, $\mathrm{H}_{0}$ was always rejected for $\mathrm{SE}$, while for DET it was accepted just once with no discard. For 2 slength, $\mathrm{H}_{0}$ was still always rejected for $\mathrm{SE}$, while for DET it was accepted in two case with no discard and in one case with discard. For 4 s-length, the null hypothesis was ac- 
Table 3. Intra-recording CV of DET and SE for 1, 2 and 4 s-length segments and the respective variation of the $\mathrm{CV}$ $(\Delta \mathrm{D})$ introduced by discarding low quality segment.

\begin{tabular}{lllllll}
\hline & 1-s & $\Delta$ D1-s & 2-s & $\Delta$ D2-s & 4-s & $\Delta$ D4-s \\
\hline \hline DET & $23.3 \%$ & $-15.6 \%$ & $19.1 \%$ & $-22.8 \%$ & $13.3 \%$ & $-47.9 \%$ \\
SE & $26.6 \%$ & $-16.1 \%$ & $20.5 \%$ & $-20.1 \%$ & $13.9 \%$ & $-42.2 \%$ \\
\hline
\end{tabular}

Table 4. Intra-patient CV of DET and SE for 1,2 and 4 s-length segments and the respective variation of the CV $(\Delta \mathrm{D})$ introduced by discarding low quality segment.

\begin{tabular}{lllllll}
\hline & 1-s & $\Delta$ D1-s & 2-s & $\Delta$ D2-s & 4-s & $\Delta$ D4-s \\
\hline \hline DET & $23.9 \%$ & $+2.0 \%$ & $24.8 \%$ & $+3.5 \%$ & $24.1 \%$ & $+7.7 \%$ \\
SE & $34.2 \%$ & $+0.5 \%$ & $34.8 \%$ & $-0.1 \%$ & $35.9 \%$ & $-0.3 \%$ \\
\hline
\end{tabular}

cepted in 7 cases ( 1 for SE with discard, 1 for DET with no discard and 5 for DET with discard), however, once visualized the box plots to verify the accuracy of the results, the median values were revealed different. The inaccuracy found for the 4 s-length datasets are justified by the fact that the sample size are so small, that the test does not follow a $\chi^{2}$ distribution.

\section{Conclusion}

The discarding process benefitted more the intrarecording stability than the intra-patient. However the remained high variability of the $\mathrm{CV}$ has revealed that averaging data in one recording, as well as among measurement sites, may lead to an unfair oversimplification of CFAEbased atrial substrate characterization. In particular, in the intra-patient analyses the visualized box plots showed many cases in which just a part of the measurement sites presented similar atrial remodeling identified by alike DET and SE values, enhancing the conclusion that averaging makes lose the singularity of substrate at the different atrial sites, which is the base of personalized treatments.

\section{Acknowledgements}

Research supported by grants DPI2017-83952-C3 from MINECO/AEI/FEDER UE，SBPLY/17/180501/000411 from JCCLM and AICO/2019/036 from GVA.

\section{References}

[1] January CT, Wann LS, Calkins H, Chen LY, Cigarroa JE, Cleveland Jr JC, Ellinor PT, Ezekowitz MD, Field ME, Fu- rie KL, Heidenreich PA, Murray KT, Shea JB, Tracy CM, Yancy CW. 2019 AHA/ACC/HRS focused update of the 2014 AHA/ACC/HRS guideline for the management of patients with atrial fibrillation: A report of the american college of cardiology/american heart association task force on clinical practice guidelines and the heart rhythm society in collaboration with the society of thoracic surgeons. Circulation 07 2019;140(2):e125-e151.

[2] Ciaccio EJ, Biviano AB, Whang W, Vest JA, Gambhir A, Einstein AJ, Garan H. Differences in repeating patterns of complex fractionated left atrial electrograms in longstanding persistent atrial fibrillation as compared with paroxysmal atrial fibrillation. Circ Arrhythm Electrophysiol Aug 2011; 4(4):470-7.

[3] Ciaccio EJ, Biviano AB, Whang W, Gambhir A, Garan H. Different characteristics of complex fractionated atrial electrograms in acute paroxysmal versus long-standing persistent atrial fibrillation. Heart Rhythm Sep 2010;7(9):1207-15.

[4] Acharya UR, Faust O, Ciaccio EJ, Koh JEW, Oh SL, Tan RS, Garan H. Application of nonlinear methods to discriminate fractionated electrograms in paroxysmal versus persistent atrial fibrillation. Comput Methods Programs Biomed Jul 2019;175:163-178.

[5] Ndrepepa G, Karch MR, Schneider MAE, Weyerbrock S, Schreieck J, Deisenhofer I, Zrenner B, Schömig A, Schmitt C. Characterization of paroxysmal and persistent atrial fibrillation in the human left atrium during initiation and sustained episodes. J Cardiovasc Electrophysiol Jun 2002;13(6):52532.

[6] Ravi KC, Krummen DE, Tran AJ, Bullinga JR, Narayan SM. Electrocardiographic measurements of regional atrial fibrillation cycle length. Pacing Clin Electrophysiol Mar 2009;32 Suppl 1:S66-71.

[7] Sanders P, Berenfeld O, Hocini M, Jaïs P, Vaidyanathan R, Hsu LF, Garrigue S, Takahashi Y, Rotter M, Sacher F, Scavée C, Ploutz-Snyder R, Jalife J, Haïssaguerre M. Spectral analysis identifies sites of high-frequency activity maintaining atrial fibrillation in humans. Circulation Aug 2005; 112(6):789-97.

[8] Alcaraz R, Abásolo D, Hornero R, Rieta JJ. Optimal parameters study for sample entropy-based atrial fibrillation organization analysis. Comput Methods Programs Biomed Jul 2010;99(1):124-32.

[9] Schinkel S, Dimigen O, Marwan N. Selection of recurrence threshold for signal detection. The European Physical Journal Special Topics 10 2008;164:15-53.

Address for correspondence:

José J. Rieta

BioMIT.org, Electronic Engineering Department, Building 7F-5

Universitat Politecnica de Valencia

Camino de Vera, s/n, 46022, Valencia, Spain

E-mail: jjrieta@upv.es 